\title{
Kinetics of Isothermal Ferritization in Spheroidal Graphite Iron*
}

\author{
By Toshiro Owadano**, Tadashi Nishimura***, \\ Hideo Miyata**** and Yoshimi Kubo*****
}

\begin{abstract}
The elementary process of ferritization in spheroidal graphite iron is known to be a diffusion controlled phenomenon, but kinetics of volumetric ferritization of the iron is left unknown. The authors investigated volumetric ferritization in isothermal annealing of spheroidal graphite iron. Ferritized fraction of matrix in isothermally annealed iron was calculated to be a function of volume fraction of graphite nodules, graphite nodule number in unit volume or unit area, diffusional growth rate of the ferrite layer and time of annealing. The mathematical expression obtained was in good accord with experimental results. The expression was also successfully used in predicting time for complete ferritization. Furthermore, growth rate constants of the ferrite layer obtained in the experiments and adoption of Johnson-Mehl's equation in isothermal ferritization were discussed.
\end{abstract}

(Received January 18, 1975)

\section{Introduction}

Spheroidal graphite iron castings are annealed and their matrices are ferritized in order to obtain high ductility. As ferritization is superposed on pearlite formation in cooling of the iron, there are two types of ferritization, direct and indirect transformations. Brown and Hawkes $^{(1)}$ reported that unstable austenite decomposed to ferrite and graphite in direct transformation, and pearlite decomposed to ferrite and graphite in indirect transformation. They investigated the growth of a ferrite shell during isothermal ferritization and concluded that ferritization was accounted for by diffusion of carbon inward through the ferrite shell

* This paper is an abridgement of papers published in Imono (J. of Japan Foundrymen's Society), 44 (1972), 666; 46 (1974), 44, 110, 834 in Japanese.

** Department of Metallurgy, Kyushu Institute of Technology, Tobata-ku, Kitakyushu 804, Japan.

*** Graduate School, Kyushu Institute of Technology, Tobata-ku, Kitakyushu 804. Present address: Chyofu Work, Kobe Steel, Ltd., Shimonoseki 752, Japan.

**** Graduate School, Kyushu Institute of Technology, Tobata-ku, Kitakyushu 804. Present address: Sakaide Work, Mitsubishi Chemical Industries, Ltd., Sakaide 762, Japan.

***** Research Center, Hitachi Metal Co., Ltd., Tobata-ku, Kitakyushu 804, Japan. surrounding a graphite nodule. It is not yet known, however, how the mechanism they postulated can explain the ferritized fraction in the iron. On the other hand, the importance of graphite nodule number in iron has been emphasized in considering the ferritized fraction in irons $s^{(2) \sim(5)}$.

In this paper the present authors investigated the effects of three main factors on the ferritized fraction in isothermally annealed spheroidal graphite iron, namely graphite nodule number, growth rate constant of the ferrite layer and time of annealing.

\section{Mathematical Consideration}

Figure 1 shows the distribution of carbon concentration during ferritization according to the concept of Brown and Hawkes ${ }^{(1)}$. If we use the following notations:

$C_{1}=$ carbon concentration in ferrite at inner surface of shell

$C_{2}=$ carbon concentration in ferrite at outer surface of shell

$C_{3}=$ carbon concentration in austenite or pearlite

$D=$ diffusion coefficient of carbon in ferrite

$R_{0}=$ inner radius of spherical ferrite shell

$R=$ outer radius of spherical ferrite shell

$t=$ time of annealing,

the growth of a ferrite shell may be expressed by

Trans. JIM

1975 Vol. 16 


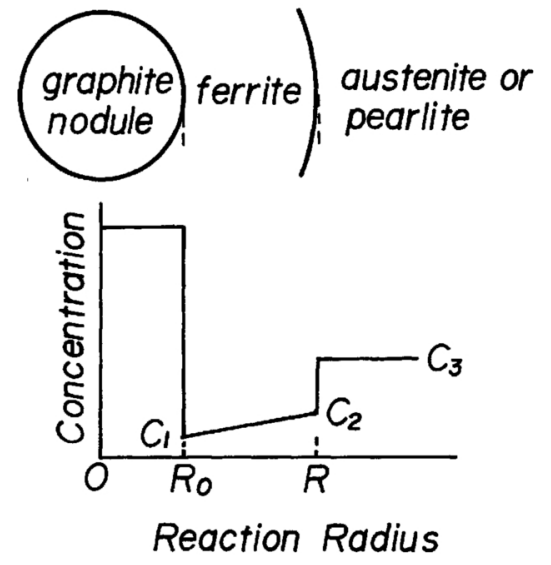

Fig. 1 Distribution of carbon concentration during ferritization.

$$
4 \pi R^{2} C_{3} d R=-4 \pi \frac{\left(C_{1}-C_{2}\right) R_{0} R}{R-R_{0}} D d t
$$

In eq. (1) the growth of the graphite nodule in the center of the ferrite shell during ferritization is neglected, and $C_{1}$ and $C_{2}$ are assumed to be neglesible in comparison with $C_{3}$. Introducing a parameter $\beta=R / R_{0}$ and integrating eq. (1) result in

$$
\frac{\left(C_{2}-C_{1}\right) D t}{C_{3} R_{0}^{2}}=\frac{1}{6}\left(2 \beta^{3}-3 \beta^{2}+1\right) .
$$

If we assume that all graphite nodules in the iron are equi-sized,

$$
V_{G}=\frac{4}{3} \pi N_{V} R_{0}^{3}
$$

where $V_{G}$ and $N_{V}$ denote volume fraction of graphite nodules in the iron and graphite nodule number in unit volume of iron, respectively. Equations (2) and (3) lead to

$$
\left(\frac{4 \pi}{3 V_{G}}\right)^{2 / 3} N_{V}{ }^{2 / 3} \frac{C_{2}-C_{1}}{C_{3}} D t=\frac{1}{6}\left(2 \beta^{3}-3 \beta^{2}+1\right) .
$$

The volume fraction of ferrite in the matrix of iron $F$ may be computed by

$$
\frac{d F}{d t}=(1-F) 4 \pi R^{2} N_{V} \frac{d R}{d t},
$$

where $1-F$ is introduced by considering mutual impingements of neighboring ferrite shells. Integration of eq. (5) under conditions of $F=0$ at $R=R_{0}$ results in

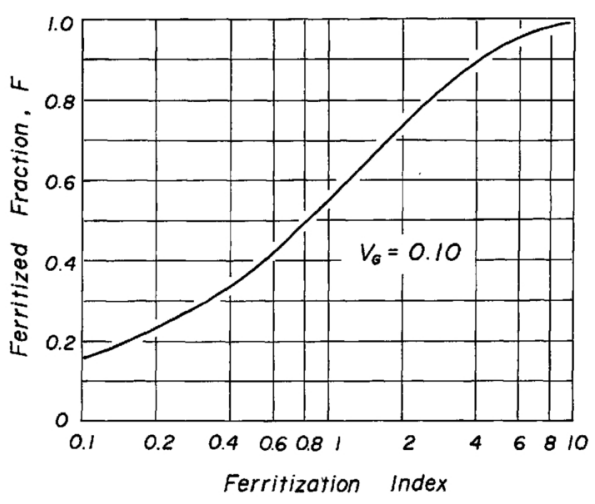

Fig. 2 Ferritized fraction as a function of ferritization index (FI) defined by eq. (7) or eq. (9).

$$
\begin{aligned}
F & =1-\exp \left[-\frac{4}{3} \pi N_{V}\left(R^{3}-R_{0}^{3}\right)\right] \\
& =1-\exp \left[-\left(\beta^{3}-1\right) V_{G}\right] .
\end{aligned}
$$

Combining eqs. (4) and (6), and using the experimental value of $V_{G}=0.10$, we can get $F$ as a unique function of "Ferritization Index" $(F I)$, as shown in Fig. 2, if we name the left term in eq. (4) ferritization index, that is,

$$
\begin{aligned}
F I & =\left(\frac{4 \pi}{3 V_{G}}\right)^{2 / 3} N_{V}{ }^{2 / 3} \frac{C_{2}-C_{1}}{C_{3}} D t \\
& =\left(\frac{4 \pi}{3 V_{G}}\right)^{2 / 3} N_{V}^{2 / 3} K t,
\end{aligned}
$$

where

$$
K=\frac{C_{2}-C_{1}}{C_{3}} D .
$$

One of the present authors reported previously that $N_{V}$ can be related to graphite nodule number in unit area $N_{A}$ by

$$
N_{V}=\left(\frac{\pi}{6 V_{G}}\right)^{1 / 2}\left(\alpha N_{A}\right)^{3 / 2},
$$

where $\alpha$ is a coefficient which adjust the unequality of nodule radii and vary in a narrow range of $1.00 \sim 1.26$ for ordinary spheroidal graphite iron ${ }^{(6)}$. Then, $F I$ is also written as

$$
F I=\frac{2 \pi}{3 V_{G}} \alpha N_{A} K t \text {. }
$$

In plastically compressed iron, graphite nodules in the iron are flattened and beside them are observed approximately one-dimensional growth of the ferrite layer during annealing. The average thickness of the ferrite 
layer $x$ is related to the time of annealing $t$ by

$$
\begin{aligned}
C_{3} d x & =\frac{C_{2}-C_{1}}{x} D d t, \text { or } \\
x^{2} & =2 K t .
\end{aligned}
$$

Thus we may call $K$ the growth rate constant of the ferrite layer.

\section{Experimental Technique}

\section{Specimens}

Chemical analyses of spheroidal graphite iron castings used are tabulated in Table 1. Small specimens with size $5 \mathrm{~mm} \times 8 \mathrm{~mm} \times 10 \mathrm{~mm}$ were prepared for ferritization annealing by sawing the castings.

\section{Graphite nodule number}

A specimen was heated at $900^{\circ} \mathrm{C}$ for $2 \mathrm{hr}$ in order to austenitize the matrix, and at $800^{\circ} \mathrm{C}$ for $1 \mathrm{hr}$ and cooled rapidly. Graphite nodule number in unit area $N_{A}$ and fractional area of graphite nodules $V_{G}$ were measured in the center of the specimen. In several specimens, frequencies of finding nodule diameters in constant size intervals were measured in order to compute the nodule number in unit volume
$N_{V}$ by means of the Schwartz-Saltykov $\operatorname{method}^{(7)}$.

\section{Ferritization}

Heat cycles for ferritization were;

(a) $900^{\circ} \mathrm{C} \times 2 \mathrm{hr} \rightarrow 800^{\circ} \mathrm{C} \times 1 \mathrm{hr} \rightarrow$ ferritization temperature for varied times $\rightarrow$ cooled in water (b) $600^{\circ} \mathrm{C} \times 10 \mathrm{~min}$ pre-heat $\rightarrow$ ferritization temperature for varied times $\rightarrow$ cooled in water

To keep ferritization temperature constant, molten aluminum or aluminum-copper eutectic alloy bath was used. Heat cycle (a) was widely used and (b) for only indirect transformation.

\section{Ferritized fraction}

Ferritized fraction $F$ was determined by measuring fractional areas, i.e.,

$$
F=\frac{f_{G+\alpha}-f_{G}}{f_{M}}
$$

where

$f_{G+\alpha}=$ fractional area of graphite and ferrite after ferritization annealing

$f_{G}=$ fractional area of graphite nodules before ferritization annealing, $f_{G}=V_{G}$

$f_{M}=$ fractional area of matrix before ferritization, $f_{M}=1-V_{G}$.

\begin{tabular}{|c|c|c|c|c|c|c|c|}
\hline \multirow{2}{*}{ Casting } & \multicolumn{6}{|c|}{ Chemical Composition (\%) } & \multirow{2}{*}{$\begin{array}{c}\text { Shape of Casting } \\
(\mathrm{mm})\end{array}$} \\
\hline & $\mathrm{C}$ & $\mathrm{Si}$ & Mn & $\mathbf{P}$ & S & Mg & \\
\hline $\mathbf{A}$ & 3.43 & 2.95 & 0.35 & 0.011 & - & 0.045 & $25 \times 40$ with riser $\times 150$ \\
\hline B & 3.43 & 2.36 & 0.33 & 0.03 & 0.008 & 0.047 & $\begin{array}{l}10 \times 30 \times 100 \\
30 \times 40 \times 100 \\
50 \times 60 \times 100\end{array}$ \\
\hline $\mathrm{C}$ & $\begin{array}{l}3.34 \\
3.41 \\
3.44 \\
3.46 \\
3.57 \\
3.37 \\
\end{array}$ & $\begin{array}{l}1.01 \\
1.59 \\
1.98 \\
2.43 \\
2.92 \\
3.60 \\
\end{array}$ & $\begin{array}{l}0.32 \\
0.32 \\
0.31 \\
0.33 \\
0.32 \\
0.31 \\
\end{array}$ & $\begin{array}{l}0.010 \\
0.010 \\
0.010 \\
0.011 \\
0.010 \\
0.010 \\
\end{array}$ & $\begin{array}{l}0.010 \\
0.009 \\
0.010 \\
0.009 \\
0.009 \\
0.008 \\
\end{array}$ & $\begin{array}{l}0.041 \\
0.038 \\
0.046 \\
0.039 \\
0.039 \\
0.042 \\
\end{array}$ & $25 \phi \times 200$ \\
\hline $\mathbf{D}$ & $\begin{array}{l}3.56 \\
3.38 \\
3.43 \\
3.62 \\
3.62 \\
3.65\end{array}$ & $\begin{array}{l}2.45 \\
2.55 \\
2.42 \\
2.51 \\
2.54 \\
2.51\end{array}$ & $\begin{array}{l}0.00 \\
0.12 \\
0.20 \\
0.30 \\
0.54 \\
0.85\end{array}$ & $\begin{array}{l}0.012 \\
0.015 \\
0.010 \\
0.013 \\
0.013 \\
0.013\end{array}$ & $\begin{array}{l}0.019 \\
0.011 \\
0.010 \\
0.015 \\
0.017 \\
0.016\end{array}$ & $\begin{array}{l}\mathbf{0 . 0 3 8} \\
\mathbf{0 . 0 5 6} \\
\mathbf{0 . 0 4 1} \\
0.054 \\
\mathbf{0 . 0 5 8} \\
\mathbf{0 . 0 4 8}\end{array}$ & $25 \phi \times 200$ \\
\hline
\end{tabular}

Although ferritized fraction $F$ and fraction of

Table 1 Chemical analyses and shape of spheroidal graphite iron castings. 


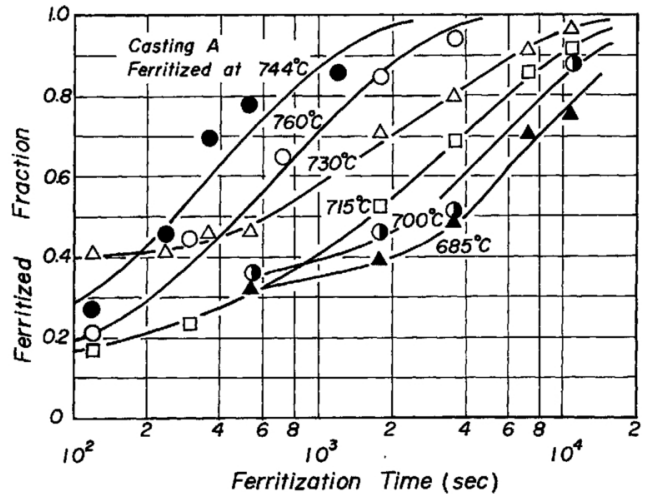

Fig. 3 Ferritized fraction in spheroidal graphite iron annealed for varied time at several temperatures after austenitizing.

graphite nodules in iron were measured in fractional area, they are equal to the volume fractions of ferrite and graphite respectively ${ }^{(8)}$.

\section{Experimental Results}

Figure 3 shows the change of ferritized fraction $F$ with time and temperature of ferritization annealing in heat cycle (a) of $A$ specimens. In ferritization at 760 and $744^{\circ} \mathrm{C}$, the microstructure of the quenched specimens was composed of graphite, ferrite and martensite, which means that ferritization by direct transformation was proceeding prior to quenching. But at $730^{\circ} \mathrm{C}$, pearlite instead of martensite appeared after about 100 -sec annealing, and afterwards indirect transformation took place.

Figure 4 shows plots of $F I$ corresponding to the measured $F$ in Fig. 3 vs time of ferritization, which are linear relations as expected in eq. (7), since $N_{V}$ and $K$ do not vary during isothermal ferritization. As the average value of the measured $V_{G}$ was 0.10 and $N_{V}$ in $A$ specimens was $3720000 / \mathrm{cm}^{3}$, slopes of straight lines in Fig. 4 enable computation of $K$, the growth rate constant of the ferrite layer, by eq. (7).

When $A$ specimens with the pearlite matrix were ferritized by heat cycle (b), indirect transformation proceeded as shown in Fig. 5. $K$ in each ferritization temperature was determined in a similar way to that shown in Fig. 4.

The values of $K$ thus determined are shown in Fig. 6 in relation to the reciplocal of ferritiza-

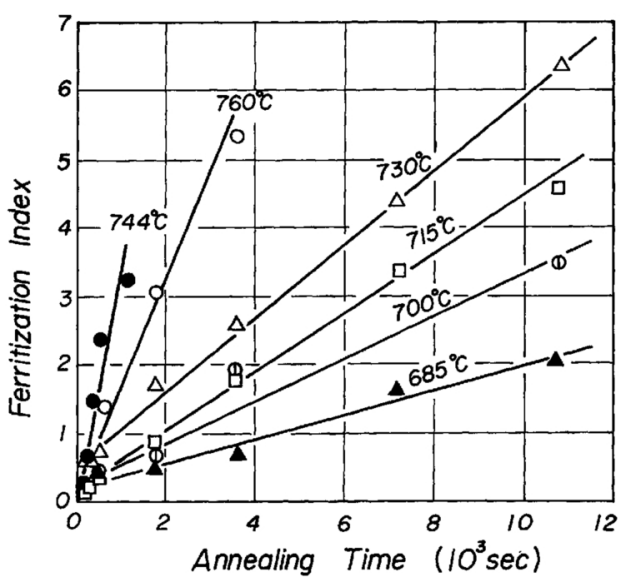

Fig. 4 Ferritization index vs annealing time plots of the data shown in Fig. 3.

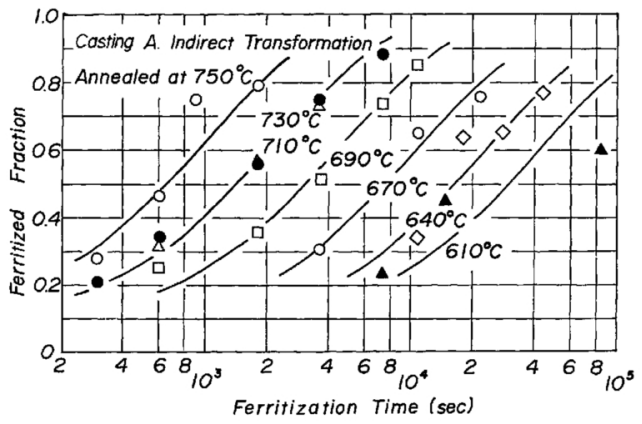

Fig. 5 Ferritized fraction in spheroidal graphite iron reheated at several temperatures for varied time.

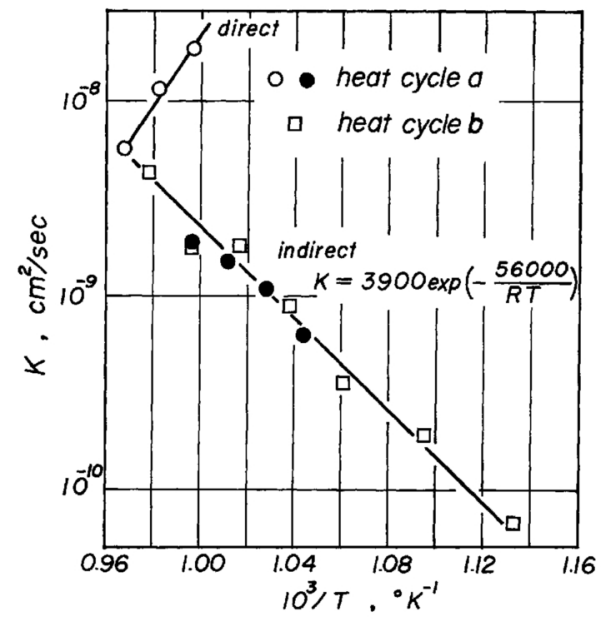

Fig. 6 Growth rate constant of ferrite layer in relation to reciprocal of annealing temperature.

tion temperature. In direct transformation, $K$ increases as the ferritization temperature de- 


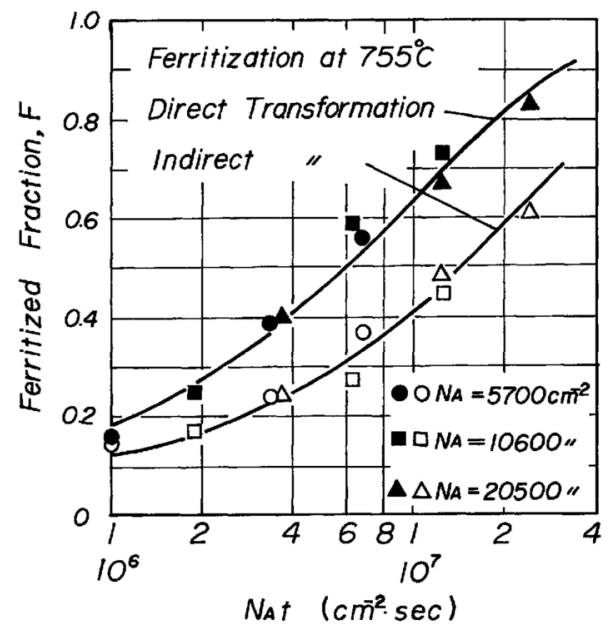

Fig. 7 Ferritized fraction vs $N_{A} t$ relation of three castings with different $N_{A}$ of the same melt (B castings).

creases, but in indirect transformation, the temperature dependency of $K$ is inverted.

$N_{A}$ and $N_{V}$ usually vary with casting dimensions of the same melt. Then check of the effect of graphite nodule number on the ferritization index may be possible by changing casting dimensions. Figure 7 shows the measured values of ferritized fraction $F$ in relation to $N_{A} t$ in $755^{\circ} \mathrm{C}$ ferritization annealing of $B$ specimens, which shows that however $N_{A}$ may vary, $F$ vs $N_{A} t$ plots fit with a curve in each case of transformation. That is, the definition of ferritization index in eq. (10) is proved to be adequate. In indirect transformation at lower temperature, however, the effect of graphite nodule number on the ferritization index decreased, when spheroidal cementite particles were prominent and the ferrite-pearlite boundary became vague in microstructure.

In Fig. 8 are shown plots of the square of the ferrite layer thickness in one-dimensional growth vs time of annealing at $730^{\circ} \mathrm{C}$ for group $C$ specimens with different silicon content. The inclination of straight lines in the figure enables us to compute $K$ by eq. (11). $K$ thus determined and $N_{A}$ in each of casting $(25 \mathrm{~mm}$ diameter rod) are shown in Fig. 9. Figure 10 is a similar one when manganese content is varied in group $D$ specimens. Both $K$ and $N_{A}$ seem to be strongly dependent on chemical compositions of the irons.

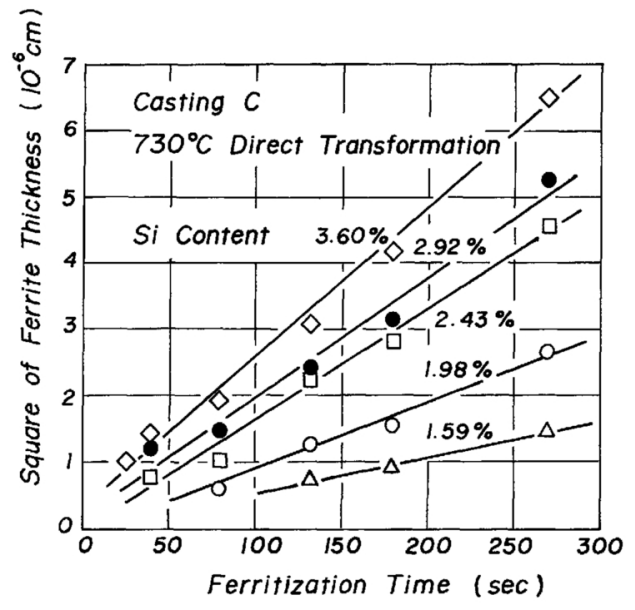

Fig. 8 One-dimensional growth of ferrite layer at $730^{\circ} \mathrm{C}$ in irons with different silicon content.

Figure 2 and eq. (10) indicate that a definite level of ferritized fraction may be obtained when $N_{A} K t$ attains a certain value which corresponds to the ferritized fraction. As an example, time for completion of ferritization is determined by microscopic observation at the same temperatures as those shown in Fig. 9 and Fig. 10 for each iron. In Fig. 11 the longest annealing time for incomplete ferritization and the shortest annealing time for complete ferritization are shown in pair in relation to $K N_{A}$. The straight line in Fig. 11 shows $K N_{A} t=0.37$ which is the calculated relation by putting $F=0.99, V_{G}=0.10$ and $\alpha=1.2$ in eqs. (4), (6) and (10). Annealing time for complete and

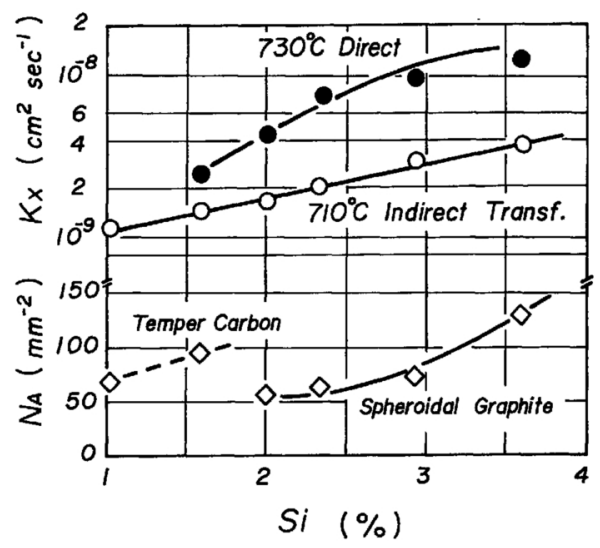

Fig. 9 Growth rate constant of ferrite layer $K$ and graphite nodule number in unit area $N_{A}$ in irons with different silicon content, diameter of rod casting $25 \mathrm{~mm}$. 


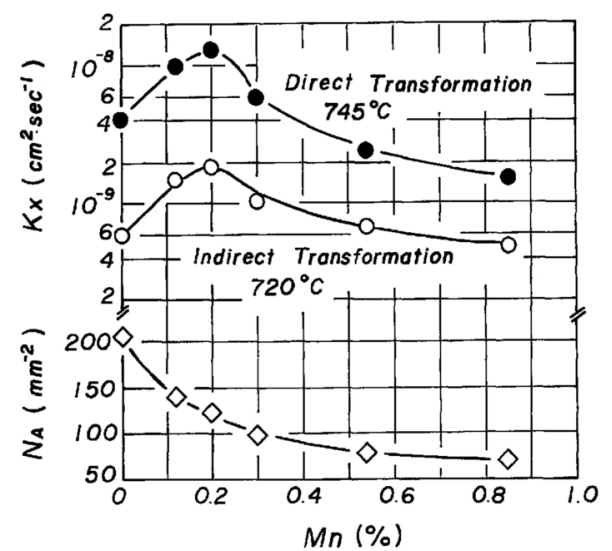

Fig. 10 Growth rate constant of ferrite layer $K$ and graphite nodule number in unit area $N_{A}$ in irons with different manganese content, diameter of rod casting $25 \mathrm{~mm}$.

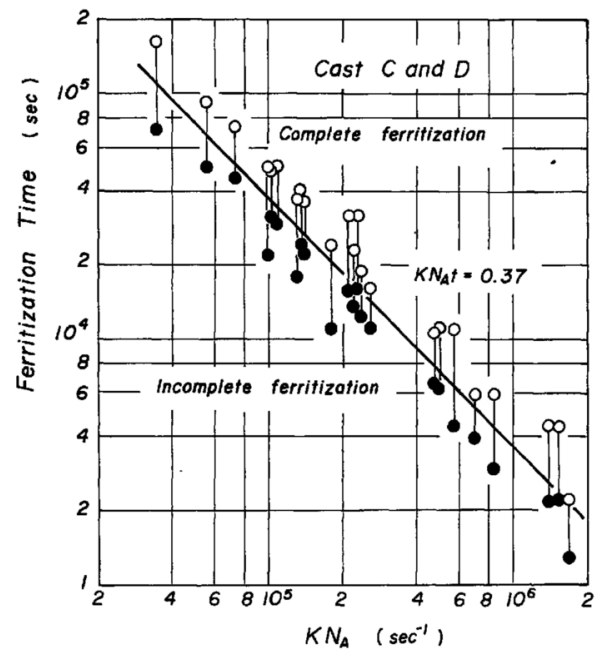

Fig. 11 Annealing time for completion of ferritization in relation to $K N_{A}$.

incomplete ferritization are well divided by the calculated straight line.

\section{Discussion}

\section{Growth rate constant of ferrite layer}

The growth rate constant of ferrite layer is defined by eq. (8). Are the measured values of $K$ reasonable? Values of $C_{2}-C_{1}$ computed from $K$ shown in Fig. 6 by putting $D=0.0038$ $\exp (-16800 / R T)^{(9)}$ and $C_{3}=0.6 \%$ carbon are shown in Fig. 12, where $R$ and $T$ denote gas

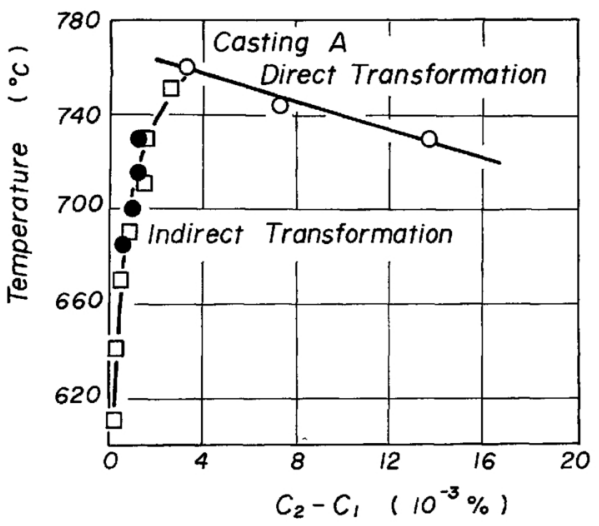

Fig. 12 Difference in carbon concentration of ferrite between outer and inner surface of shell $C_{2}-C_{1}$ calculated from experimental values of $K$.

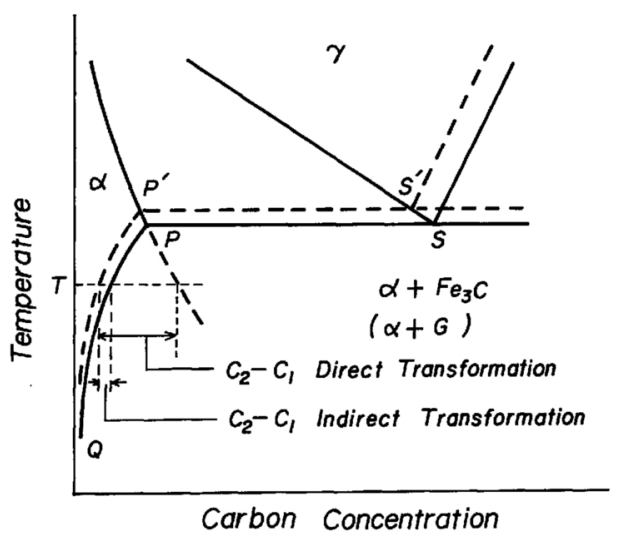

Fig. 13 Schematic $\mathrm{Fe}-\mathrm{C}$ double diagram representing the difference in carbon consentration of ferrite $C_{2}-C_{1}$ at temperature $T$.

constant and absolute temperature of ferritization annealing, respectively. The temperature dependency and the order of magnitude of $C_{2}-C_{1}$ seem to be in good accord with the concept of Brown and Hawkes ${ }^{(1)}$ represented in Fig. 13.

Addition of alloying elements such as silicon and manganese in iron may vary the values of $C_{2}-C_{1}, C_{3}$ and $D$ at the same temperature, which may be the reason why $K$ varies with silicon or manganese content as shown in Fig. 9 and Fig. 10.

\section{Johnson-Mehl's equation}

Hitherto we considered ferritization in spheroidal graphite iron to be a diffusion controlled 


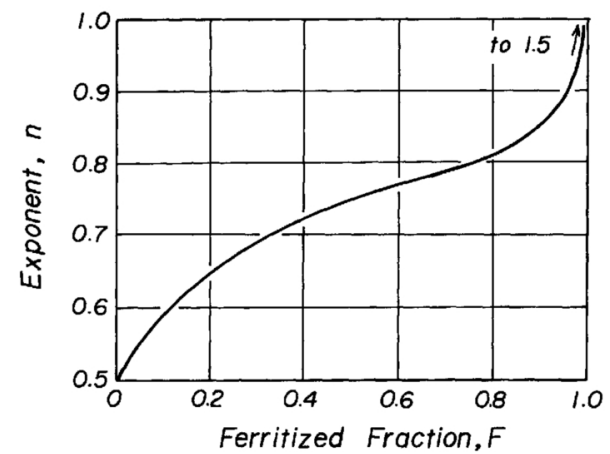

Fig. 14 Time exponent $n$ calculated as a function of ferritized fraction $F$ in isothermal annealing.

phenomenon of carbon onto definite number of graphite nodules. Then it may be of use to consider how Johnson-Mehl's equation is applicable in isothermal ferritization. In order to determine time exponent $n$ in Johnson-Mehl's equation

$$
F=1-\exp \left(-k t^{n}\right)
$$

where $k$ is a constant, $\log \log [1 /(1-F)]$ vs $\log (F I)$ plots are made by using the $F-F I$ relation shown in Fig. 2. Values of $n$ thus determined are shown in Fig. 14 in relation to $F$. Figure 14 shows that $n$ is not constant but varies with $F$, that is, when $F$ is very small, $n$ approximates to 0.5 , the value expected in one dimensional diffusion controlled process, and when $F$ approaches to 1, $n$ increases sharply to 1.5 , the value expected in spherical diffusion controlled process onto a definite number of nuclei. But in ordinary magnitude of $F$ which we determine by microscopic observation, mean value of $n$ should be about 0.75 as shown in Fig. 14, which is in very good accord with the experimentally determined values of $n=0.7 \sim$ 0.8 .

\section{Conclusions}

Ferritized fraction in isothermally annealed spheroidal graphite iron was calculated to be a function of ferritization index which was defined in terms of volume fraction of graphite nodules, graphite nodule number in unit volume or in unit area, growth rate constant of ferrite layer and time of annealing. Experimental results showed quantitative validity of defining the ferritization index and its relation to the ferritized fraction.

The silicon or manganese content in iron altered the graphite nodule number as well as the growth rate constant of the ferrite layer, which in turn may affect the time of annealing for a definite ferritized fraction. Accordingly, the annealing time for complete ferritization, for instance, was predictable when the growth rate constant of the ferrite layer and the graphite nodule number in unit area of the iron were known.

The growth rate constant of the ferrite layer determined in the present work had a reasonable order of magnitude and temperature dependency to consider the growth of ferrite diffusion controlled. And when JohnsonMehl's equation was applied in isothermal ferritization, time exponent $n$ was about 0.75 .

\section{Acknowledgments}

The authors wish to acknowledge S. Morimoto and Y. Iwabe, bachelors of Kyushu Institute of Technology, for their assistance in the experiments. They also appreciate discussion held on our work by Dr. R. Takahashi, chief engineer in Tobata Factory, Hitach Metal Co. Ltd.

\section{REFERENCES}

(1) B. F. Brown and M. F. Hawkes: Trans. A.F.S., 59 (1951), 181.

(2) S. A. Saltykov: Metallurg., 14 (1939) No. 8, 10.

(3) G. Sandoz: Trans. A.F.S., 70 (1962), 352.

(4) H. A. Schwartz and M. K. Barnet: Trans. A.F.S., 27 (1939), 570.

(5) M. J. Lalich and C. R. Loper: Trans. A.F.S., 80 (1973), 238.

(6) T. Owadano: Imono (Jurnal of Japan Foundrymen's Society) 45 (1973), 193.

(7) R. T. DeHoff and F. N. Rhines: Quantitative Microscopy, McGraw-Hill Book Co. Ltd. N.Y., (1968), p. 162.

(8) ibid., p. 46.

(9) J. K. Stanley: Phys. Rev., 75 (1949), 1627. 